\title{
A multicenter prospective cohort study to develop frailty-based prognostic criteria in heart failure patients (FLAGSHIP): rationale and design
}

Sumio Yamada ${ }^{1 *}$ D, Takuji Adachi ${ }^{2}$, Hideo Izawa ${ }^{3}$, Toyoaki Murohara ${ }^{4}$, Takaaki Kondo ${ }^{1}$ and on behalf of the FLAGSHIP collaborators

\begin{abstract}
Background: Heart failure (HF) and frailty often co-exist, and frailty in HF results in a poor prognosis. However, in Asian populations, prognostic criteria are needed to examine the effect of frailty on HF. Therefore, we conducted a nationwide cohort study to develop frailty-based prognostic criteria in HF patients (FLAGSHIP). FLAGSHIP mainly aims to 1) develop the frailty criteria based on HF-specific outcomes, 2) propose a hypothesis of the potential mechanisms of frailty manifestations in HF, and 3) examine the effects of outpatient cardiac rehabilitation on frailty.

Methods: In this prospective study, we consecutively enroll ambulatory patients admitted because of acute HF or exacerbation of $\mathrm{HF}$ and elderly patients admitted for acute myocardial infarction (age $\geq 70$ years). They will be followed up for 2 years to assess frailty and hard clinical events. The primary endpoints of FLAGSHIP are cardiac events including cardiac mortality and HF-related readmission after discharge. Secondary endpoints are readmissions because of fracture or pneumonia and all-cause mortality. We used clinical data, including the items related to the frailty phenotype to develop diagnostic criteria for frailty and known prognostic factors of HF. Cognitive function, depression, and anorexia are also considered as potential components of frailty. As of March 2018, 2650 patients (85\% was patients admitted for HF) have been registered from 30 collaborating hospitals nationwide in Japan.

Discussion: FLAGSHIP provides diagnostic criteria and fundamental information on frailty manifestations to develop the best practices for the long-term management of HF. Diagnostic criteria on frailty developed by FLAGSHIP is expected to become a novel indicator for the stratification of patients at risk to functional decline after medical or surgical treatment, and in turn to contribute to the best practices in the long-term management of HF.
\end{abstract}

Keywords: Heart failure, Frailty, Diagnostic criteria, Multicenter cohort study, Japan

\section{Background}

Frailty is a condition characterized by a decline in physiological reserve that is associated with an increased risk of adverse health outcomes when exposed to a stressor [1]. Frailty is considered a medical syndrome in elderly people, which can be improved or attenuated by interventions [1]. However, despite knowing the prognostic value of frailty, diagnostic criteria for frailty have not

\footnotetext{
* Correspondence: yamadas@met.nagoya-u.ac.jp

${ }^{1}$ Department of Health Sciences, Nagoya University Graduate School of Medicine, 1-1-20 Daiko-minami, Higashi-ku, Nagoya 461-8673, Japan Full list of author information is available at the end of the article
}

been established in Asian populations. The Cardiovascular Health Study frailty index is a widely used frailty phenotype model developed based on studies of the communitydwelling elderly, almost all of whom were Caucasian or African American [2]. Accordingly, the cut-off values for grip strength to diagnose weakness cannot be applied to Asians because of differences in physique. Recently, the Asian Working Group of Sarcopenia has proposed a new cut-off value for grip strength to diagnose decreased muscle strength in the general Asian population, which was based on an expert consensus [3]. The working group also proposed to develop new cut-off values for each item

(c) The Author(s). 2018 Open Access This article is distributed under the terms of the Creative Commons Attribution 4.0 International License (http://creativecommons.org/licenses/by/4.0/), which permits unrestricted use, distribution, and reproduction in any medium, provided you give appropriate credit to the original author(s) and the source, provide a link to the Creative Commons license, and indicate if changes were made. The Creative Commons Public Domain Dedication waiver (http://creativecommons.org/publicdomain/zero/1.0/) applies to the data made available in this article, unless otherwise stated. 
in the frailty index based on longitudinal outcome-based studies [3].

Meanwhile, there is a growing interest in frailty accompanied by heart failure (HF). We previously reported the results of a secondary analysis that suggested the possibility of frailty as a clinical marker for the management of HF patients [4], although there were several study limitations, including small sample size and confounding factors. Recent similar studies have also described a relationship between frailty and mortality $[5,6]$. Yet, further robust evidence is required to establish the prognostic impact of frailty in patients with HF in Asian populations. Moreover, most previous studies have defined frailty in HF patients based on the frailty criteria developed among healthy community-dwelling elderly and not as a disease-specific outcome of HF.

In patients with HF, sarcopenia because of aging or cachexia, or both, does exist [7]. In a conceptual model, cachexia is proposed to be a wasting syndrome characterized by the loss of muscle and adipose tissue resulting from anorexia, chronic inflammation, insulin resistance, and hypogonadism [8]. Bacterial translocation caused by increased gut permeability is one of the major potential mechanisms underlying cardiac cachexia [9]. However, this hypothesis has been presented based on evidence in patients with HF and reduced ejection fractions (HFrEF). In consideration of the differences in pathology between HFrEF and HF with a preserved ejection fraction (HFpEF), frailty may manifest under a different mechanism, according to the HF subtype. Additionally, mental health problems, such as cognitive impairment and depression, may possibly augment frailty in HF patients [10]. Therefore, frailty in patients with HF should be discussed in a multidimensional framework.

The diagnostic criteria for frailty based on HF-specific outcomes are expected to serve as standard clinical indicators in HF management. Therefore, we conducted a nationwide, multi-center, prospective cohort study to develop the frailty-based prognostic criteria in heart failure patients (FLAGSHIP).

\section{Methods/design}

\section{Study design}

FLAGSHIP is an ongoing multi-center, prospective cohort study in Japan. The study patients are enrolled during hospitalizations for $\mathrm{HF}$ and are followed-up for 2 years after discharge. This study was designed based on the Strengthening the Reporting of Observational Studies in Epidemiology (STROBE) statement.

\section{Study objectives}

The main objectives of FLAGSHIP include the following: 1) to develop the frailty criteria based on HF-specific clinical outcomes, 2) to propose a hypothesis regarding potential mechanisms of frailty manifestations in patients with $\mathrm{HF}$, and 3) to examine the effect of outpatient cardiac rehabilitation on frailty in patients with HF.

\section{Study hospitals}

The participating study hospitals were selected across Japan considering their geographical distribution (Fig. 1). All the participating hospitals provided standardized cardiac inpatient rehabilitation services according to the Japanese Association of Cardiac Rehabilitation. Very few hospitals do not provide cardiac outpatient rehabilitation services.

\section{Study population}

Eligibility criteria for FLAGSHIP include 1) ambulatory patients admitted due to $\mathrm{HF}$, and 2) ambulatory patients aged 70 or over, admitted because of acute myocardial infarction (AMI). Ambulatory patients are defined as those capable of walking $20 \mathrm{~m}$ at the time of discharge, with or without assistance or walking aids. The exclusion criteria included the presence of one or more of the following: 1) severe cognitive impairment defined by a score on the Mini-Mental State Examination (MMSE) [11] < 17 points [12], 2) severe mental disorder, 3) difficulty in answering questionnaires, and 4) an assumed impending mortality (e.g., severe aortic valve stenosis not amenable to surgical intervention, terminal stage cancer). Patients readmitted to the hospital during the study period are enrolled at the time of the first hospitalization.

\section{Sample size calculation}

The sample size was calculated by performing a multivariate analysis, which examined the relationship between measured frailty items and cardiac events, a primary endpoint in this study. In the multivariate analysis, 30 of the independent variables, including frailty items and known prognostic factors of $\mathrm{HF}$, were selected for the analysis. Assuming 10 outcomes per one independent variable, we needed to observe 300 outcomes. Based on our preliminary data, a $20 \%$ incidence rate of a cardiac event in 2 years was estimated. With a $15 \%$ estimated drop-out rate, the necessary sample size was calculated to be 1764 patients. To perform the multivariate analysis stratified by HFrEF and HFpEF, the final target sample size was determined to be 3500 .

\section{Data collection and processing}

The study protocol is shown in Fig. 2. Development of the frailty criteria in HF, a primary objective of this study, is conducted using baseline data and subsequent follow-up surveys. Cox proportional hazards model is used to assess the independent relationship between each frailty item and study endpoints adjusted for conventional prognostic factors in HF. Then, receiver operating characteristic 


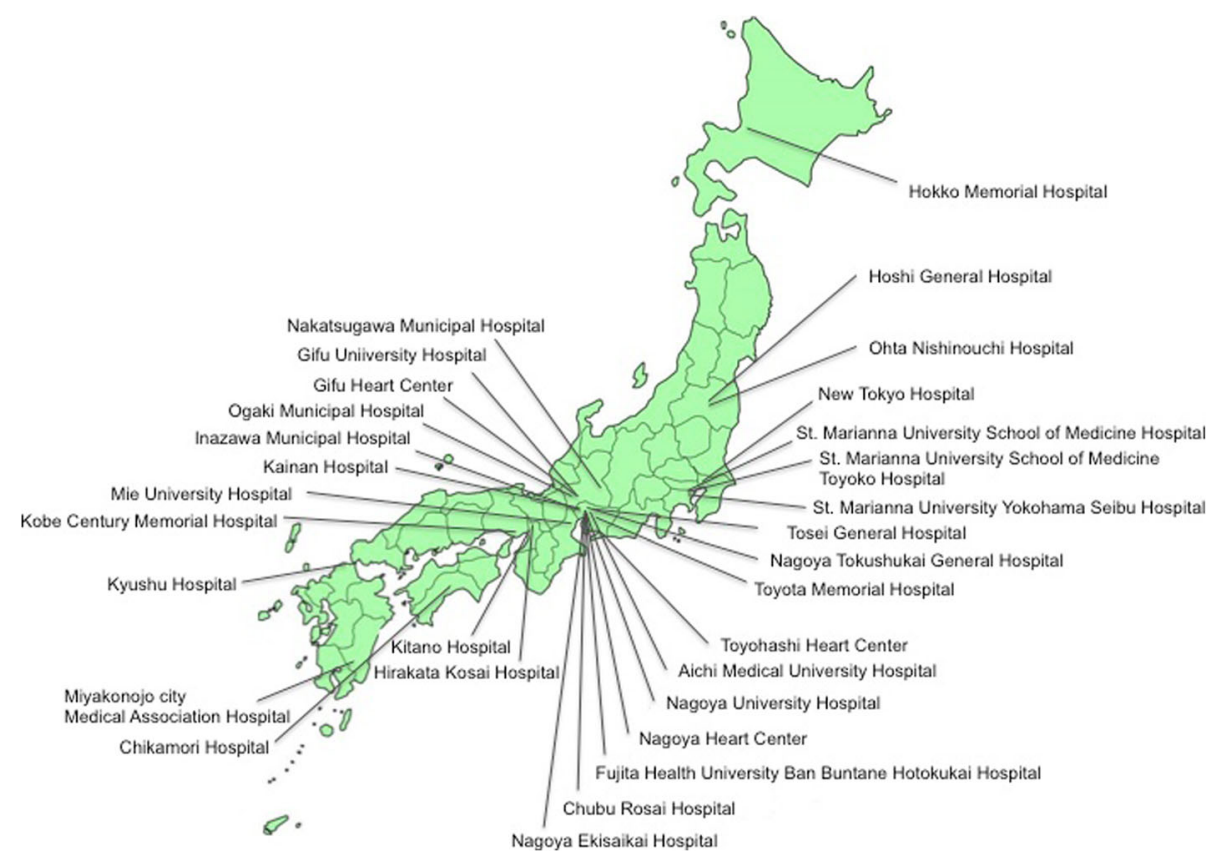

Fig. 1 Collaborating hospitals

analysis is performed to identify a cut-off value of each frailty item to predict study endpoint. Because prognostic factors in HF are likely to differ by short-term and long-term outcomes, we assess prognostic impact of frailty on 6-month and 2-year prognosis. Study endpoints and frailty assessment are as described below.

In advance of this longitudinal analysis, a cross-sectional analysis of the baseline data is performed to examine the determinants of frailty in HFrEF and HFpEF and to propose a hypothesis for frailty manifestations in HF. Additionally, the effects of outpatient cardiac rehabilitation on frailty are examined in a non-randomized study.
Any changes in the indicators of frailty from 1 to 6-months after discharge are compared between those who did and those who did not participate in cardiac rehabilitation after discharge. Propensity score matching is conducted to control for the influence of potential confounding factors.

The collaborating hospitals are encouraged to register the patients as consecutively as possible. Once informed consent is obtained, the data center assigns a study ID to the patient for linkable anonymizing. Next, data are registered via the website designated for the FLAGSHIP study. For security reasons, unique IDs and passwords are assigned

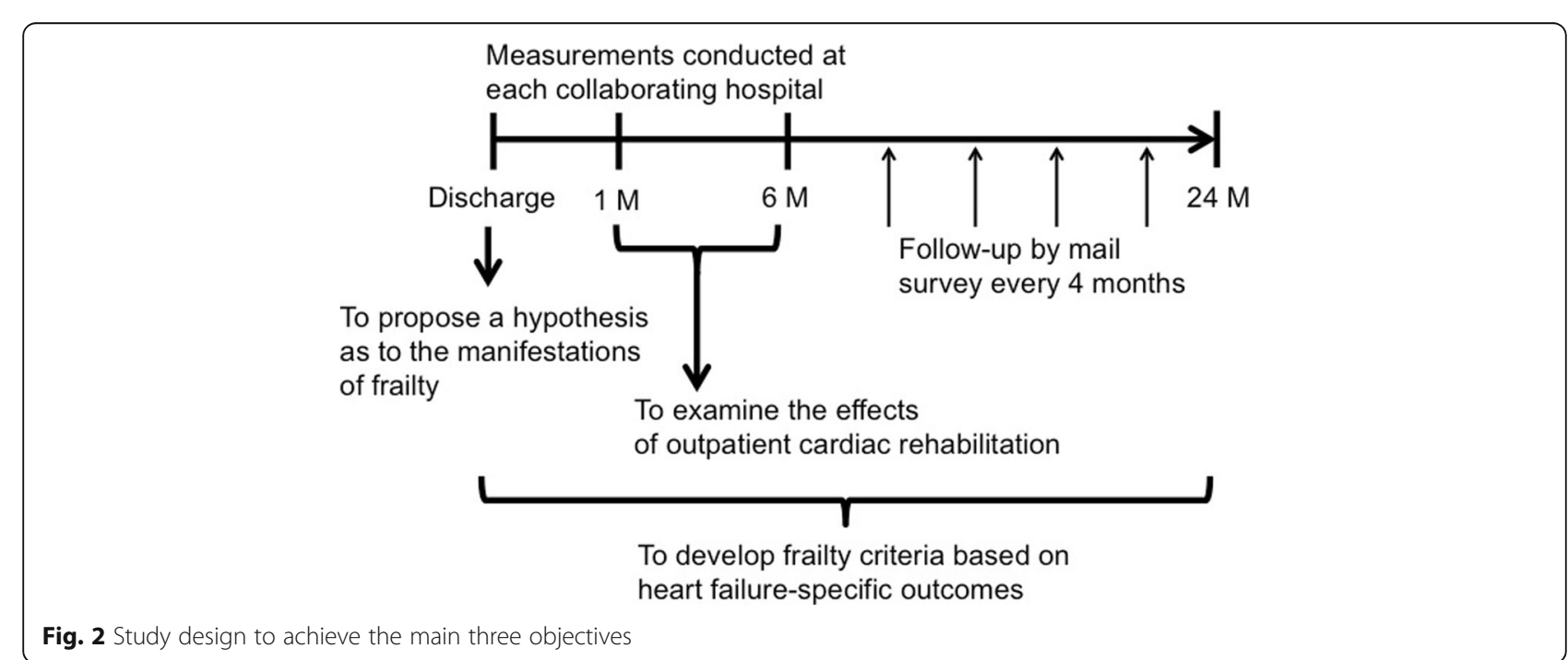


for each hospital to use the electronic system. The website is encrypted using secure sockets layer, and our data server is protected by a robust firewall to prevent unauthorized access to any information that we store (Fig. 3).

For each patient, the demographic data, etiologies of $\mathrm{HF}$, precipitating causes, comorbidities, laboratory assessments, echocardiographic findings, medications, and frailty assessments during the hospitalization are collected. At 1 month after discharge, a frailty assessment is performed again. At 6 months, data on laboratory measurements, echocardiographic findings, medications, and frailty are obtained if the patients received outpatient treatment from the collaborating hospitals after discharge. Follow-up mail surveys are sent after 6 months for each patient, and then re-sent every 4 months until 24 months after discharge. The mail survey includes outcome surveys and frailty questionnaires. Frailty questionnaires consist of exhaustion, physical inactivity and appetite loss. Body weight at each follow-up point is also collected. Selfmeasurement of body weight at home is performed using the digital weight scale prepared by research office (HD-661, TANITA Corporation, Japan). Trajectories of each frailty questionnaire and body weight after discharge are analyzed using the group-based trajectory modeling as a sub-study of FLAGSHIP.

The primary endpoints of FLAGSHIP are cardiac events including cardiac mortality and HF-related readmission after discharge. Secondary endpoints are readmissions because of fracture or pneumonia and all-cause mortality.

\section{Frailty assessment}

The frailty assessment in this study includes several aspects of frailty, in consideration of the pathophysiology of HF.
Weight loss is assessed by the body mass index (BMI). In general, unintentional weight loss in the prior 6 months is considered in the assessment of weight loss with respect to the frailty criteria. However, the mean age of Japanese patients with HF is approximately 70 years, and cognitive decline is often observed. Therefore, we selected the BMI as an objective indicator for defining weight loss instead of using self-reported assessments.

Slowness and weakness are assessed by the 10-m usual walking speed (UWS) and grip strength (GS), respectively. All measurements of grip strength are performed using the Jamar dynamometer (Digital Hand Dynamometer, DHD-1, SAEHAN Corporation, South Korea) set at the second handle position. The participants sat with the wrist in a neutral position and the elbow flexed at $90^{\circ}$. Before starting the enrollment of the study patients, measurement reliabilities for UWS and GS were confirmed at each hospital. The examiners, typically two to four physical therapists per hospital, measured UWS and GS twice each on five in-patients, aged approximately 70 years, on different days. Each subject was also measured by the other examiners. From that data, the intraclass and interclass correlation coefficients were calculated. Each hospital started to enroll the patients after providing $>0.9$ of intraclass and interclass correlation coefficients.

Exhaustion is assessed using the Performance Measure of Activity in Daily Living-8 (PMADL-8) [13]. PMADL-8 is a questionnaire assessing functional limitations. It comprises a list of eight items potentially requiring daily physical activity in chronic heart failure by a four-category response scale. It is scored from 8 to 32, with higher scores indicating more severe functional limitations. The score is strongly and negatively correlated to the peak

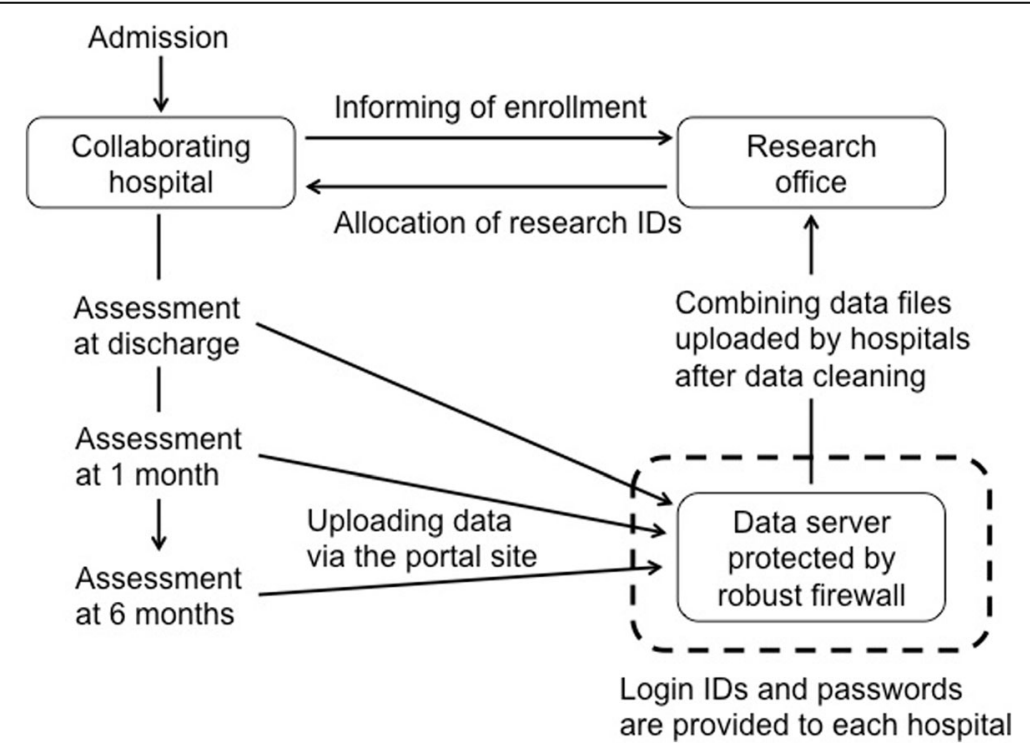

Fig. 3 Data registration system and security for data protection 
$\mathrm{VO}_{2}$ measured by the cardiopulmonary exercise test [14]. Reliability and validity of the PMADL- 8 has been published elsewhere [13, 15].

Physical inactivity is assessed using a questionnaire composed of seven items with a five-point Likert scale, in consideration of clinical utility. The total score (7-35 points) has a moderate to strong correlation with step counts and moderate to vigorous physical activity and is objectively measured by an electrical accelerometer in patients after a mild stroke or with a history of cardiac disease. The validation study of this questionnaire has now been submitted.

In addition to the aforementioned physical aspects, appetite, cognitive function, and depression are also assessed as components of frailty. Appetite is assessed using Simplified Nutritional Appetite Scale (SNAQ) [16] composed of a list of four items with a four-point Likert scale, the higher scores indicate better appetites. The score of the SNAQ (4-20) predicts weight loss in the subsequent 6 months to 1 year in elderly people.

Cognitive function is assessed using an MMSE, which is a standard test to assess global cognitive function, and includes 11 questions with a maximum score of 30 [11]. Depression is assessed using a five-item geriatric depression scale (GDS5) questionnaire, and a score $\geq 2$ points was defined as depression [17].

\section{Patient confidentiality}

The study protocol of FLAGSHIP was organized to according to the Guidelines for the Epidemiological Research proposed by the Japanese Ministry of Health, Labour and Welfare. Additionally, the study protocol was approved by the ethics committee of Nagoya University School of Medicine (approval no. 2014-0421). Ethical approval was also obtained from each participating hospital (Additional file 1), and each patient provided written informed consent to be registered to this study. Treatment methods and hospital care for the patients are not altered due to participation in this study.

\section{Discussion}

Frailty coexisting with HF is concerning in Japan as well as in Western countries because of the increasing number of patients that are living longer. To our knowledge, FLAGSHIP is the first large-scale, prospective, multicenter cohort study to develop diagnostic criteria for frailty based on HF-specific outcomes worldwide. This cohort study was also designed to propose a hypothesis regarding the potential mechanisms of frailty manifestations and to examine the effect of outpatient cardiac rehabilitation on frailty in HF. By providing these fundamental frailty data, FLAGSHIP contributes to guiding best practices for the long-term management of HF.
The prognostic impact of frailty on HF has recently been documented in several publications [4-6, 18]. We have also published the results of the secondary analysis regarding the relationship between frailty and the increased risk of cardiac events and mortality in HF [4]. However, diagnostic criteria for frailty, including the appropriate frailty items and the respective cut-off values to predict disease-specific outcomes, remain to be established. To this end, we conducted this nationwide, prospective cohort study. In this study, several aspects of the fragile state of patients were assessed. We observed physical function, appetite, and psychophysiological function in consideration of the possible mechanisms of frailty manifestations in HF. An appropriate set of frailty items and their cut-off values will be determined in the future using disease-specific outcomes and follow-up data. A trajectory analysis will also facilitate the analysis of post-discharge time-related trends in body weight and functional limitations, as well as appetite loss.

In $\mathrm{HF}$, cachexia, a wasting syndrome characterized by the loss of muscle and adipose tissue [8], causes secondary sarcopenia [7], and in turn frailty. Anorexia is one of the main causes of cachexia [8], and results from intestinal ischemia or congestion, and systemic inflammation [19]. Hence, evaluating anorexia may be helpful in assessing frailty due to a cachectic state, and it is considered a candidate component of HF-induced frailty. Depression that also often coexists in HF [10] is another candidate to be potentially included for defining frailty. We previously reported that depression in HF was associated with severe functional limitations after discharge [20]. Additionally, a previous meta-analysis of the community-dwelling elderly demonstrated a bilateral relationship between depression and frailty [21]. Based on these findings, we included appetite and psychophysiological function in the frailty assessment, in addition to physical components proposed as part of the frailty phenotype.

FLAGSHIP also included patients aged $\geq 70$ years admitted because of AMI even if they did not manifest HF. Older age is one of the major risk factors for HF [22], which may relate to frailty or mortality following AMI. Preventive management of HF, therefore, should be considered for the clinical management of the elderly with AMI. Walking speed, a major phenotype of frailty, has been reported to predict cardiovascular events after AMI [23]. In addition, a recent cohort study demonstrated a relationship between geriatric condition and poor prognosis following acute coronary syndrome [24]. From these evidences, FLAGSHIP also aimed to examine the prevalence of frailty and its trajectory in aged AMI.

Each patient enrolled into this study will be followed-up for 2 years after discharge. In our previous report [4],36 out of 181 patients (19.9\%) with a mean age of $68.1 \pm$ 9.7 years (who were younger than the patients generally 
seen in actual clinical practice), experienced cardiac death or a HF readmission during the 2 years after discharge. Additionally, another of our previous studies in the elderly with care needs demonstrated that $>25 \%$ of the participants experienced disability progression during the 3 years observation [25]. Based on these preliminary observations, we determined that 2 years of follow-up for FLAGSHIP was sufficient to observe study outcomes. In addition to the disease-specific outcome, readmissions due to fracture or pneumonia were frequently observed events in the fragile elderly, and are also considered to be notable outcomes in FLAGSHIP. It is easily assumed that deconditioning after fracture or pneumonia will result in the progression of frailty. In addition, systemic inflammation due to pneumonia or fracture is likely to make HF management more difficult $[26,27]$. FLAGSHIP is therefore designed to examine frailty-induced clinical outcomes. Another aim of this cohort study was to examine the effects of cardiac rehabilitation on frailty. Patients are referred for cardiac rehabilitation in daily practice to provide high-quality long-term management. Exercise programs in cardiac rehabilitation have favorable effects on HF prognosis. However, there is limited evidence of the effects of cardiac rehabilitation among HF patients with frailty or sarcopenia, because such patients are often excluded from clinical trials. Observational results with a larger study sample size will allow us to analyze the effects of cardiac rehabilitation on frailty in real-world clinical practice. Along with these, many sub-studies are currently going beside the main stream, for instance, hypothesis generation to become a fragile based on HF subtype, etc. Future reports from FLAGSHIP will continue to provide clinical information that will be of value in clinical practice.

\section{Conclusions}

Diagnostic criteria on frailty developed by FLAGSHIP is expected to become a novel indicator for the stratification of patients at risk to functional decline after medical or surgical treatment, and in turn to contribute to the best practices in the long-term management of HF.

\section{Additional files}

Additional file 1: Hospitals and Ethics Committees enrolled in the FLAGSHIP study. (PDF $136 \mathrm{~kb}$ )

Additional file 2: FLAGSHIP investigators. (PDF 8 kb)

\section{Abbreviations}

AMI: Acute myocardial infarction; BMI: Body mass index; GDS5: 5-item Geriatric Depression Scale; GS: Grip strength; HF: Heart failure; HFpEF: Heart failure with preserved ejection fraction; HFrEF: Heart failure with reduced ejection fraction; MMSE: Mini-Mental State Examination; PMADL-8: Performance Measure of Activity in Daily Living-8; SNAQ: Simplified Nutritional Appetite Scale; UWS: Usual walking speed

\section{Acknowledgements}

We are grateful to all of the patients for cooperation with the study and also thank all collaborating investigators for their contributions. The FLAGSHIP investigators are listed in Additional file 2.

Availability of data and material

Not applicable.

\section{Funding}

This study is supported by a Grant-in-Aid for Scientific Research (A) from the Japan Society for the Promotion of Science [16H01862]. We did not receive any funding support from commercial organization for this study.

\section{Authors' contributions}

SY is a principal investigator and contributed to protocol development and drafted this manuscript. TA is an investigator and contributed to the study design and protocol development and drafted this manuscript. $\mathrm{HI}$ is an investigator and contributed to the study design and protocol development. $\mathrm{TM}$ is an investigator and contributed to the study design and protocol development. TK is an investigator and contributed to the study design and protocol development. All authors critically reviewed and edited this manuscript and approved the final protocol.

\section{Ethics approval and consent to participate}

The study protocol of FLAGSHIP was organized according to the Guidelines for the Epidemiological Research proposed by the Japanese Ministry of Health, Labour and Welfare. Additionally, the study protocol was approved by the ethics committee of Nagoya University School of Medicine (approval no. 2014-0421). Ethical approval was also obtained from each participating hospital (Additional file 1), and each patient provided written informed consent to be registered to this study. Treatment methods and hospital care for the patients are not altered due to participation in this study.

Consent for publication

Not applicable.

\section{Competing interests}

No conflict of interest declared.

\section{Publisher's Note}

Springer Nature remains neutral with regard to jurisdictional claims in published maps and institutional affiliations.

\section{Author details}

${ }^{1}$ Department of Health Sciences, Nagoya University Graduate School of Medicine, 1-1-20 Daiko-minami, Higashi-ku, Nagoya 461-8673, Japan.

${ }^{2}$ Program in Physical and Occupational Therapy, Nagoya University Graduate School of Medicine, 1-1-20 Daiko-minami, Higashi-ku, Nagoya 461-8673, Japan. ${ }^{3}$ Department of Cardiology, Fujita Health University Banbuntane Hotokukai Hospital, 3-6-10 Otobashi, Nakagawa-ku, Nagoya 454-8509, Japan. ${ }^{4}$ Department of Cardiology, Nagoya University Graduate School of Medicine, 65 Tsurumai-cho, Showa-ku, Nagoya 466-8550, Japan.

Received: 25 June 2018 Accepted: 25 July 2018

Published online: 02 August 2018

References

1. Morley JE, Vellas B, Abellan van Kan G, et al. Frailty Consensus: A Call to Action. J Am Med Dir Assoc. 2013;14(6):392-7. https://doi.org/10.1016/j. jamda.2013.03.022

2. Fried LP, Tangen CM, Walston J, et al. Frailty in older adults: evidence for a phenotype. J Gerontol A Biol Sci Med Sci. 2001;56(3):M146-56. https://doi. org/10.1093/gerona/56.3.M146.

3. Chen LK, Liu LK, Woo J, et al. Sarcopenia in Asia: consensus report of the Asian working Group for Sarcopenia. J Am Med Dir Assoc. 2014;15(2):95-101. https://doi.org/10.1016/j.jamda.2013.11.025.

4. Yamada S, Kamiya K, Kono Y. Frailty may be a risk marker for adverse outcome in patients with congestive heart failure. ESC Hear Fail. 2015;2(3): 168-70. https://doi.org/10.1002/ehf2.12052. 
5. McNallan SM, Chamberlain AM, Gerber Y, et al. Measuring frailty in heart failure: a community perspective. Am Heart J. 2013;166(4):768-74. https:// doi.org/10.1016/j.ahj.2013.07.008.

6. Vidan MT, Blaya-Novakova V, Sanchez E, Ortiz J, Serra-Rexach JA, Bueno H. Prevalence and prognostic impact of frailty and its components in nondependent elderly patients with heart failure. Eur J Heart Fail. 2016:869-75. https://doi.org/10.1002/ejhf.518.

7. Cruz-Jentoft AJ, Baeyens JP, Bauer JM, et al. Sarcopenia: European consensus on definition and diagnosis: report of the European working group on sarcopenia in older people. Age Ageing. 2010;39(4):412-23. https://doi.org/10.1093/ageing/afq034.

8. Evans WJ, Morley JE, Argilés J, et al. Cachexia: a new definition. Clin Nutr 2008;27(6):793-9. https://doi.org/10.1016/j.clnu.2008.06.013.

9. Anker SD, von Haehling S. Inflammatory mediators in chronic heart failure: an overview. Heart. 2004;90(4):464-70. https://doi.org/10.1136/hrt.2002.007005.

10. Vogels RL, Scheltens $P$, Schroeder-Tanka JM, Weinstein HC. Cognitive impairment in heart failure: a systematic review of the literature. Eur J Heart Fail. 2007;9(5):440-9. https://doi.org/10.1016/j.ejheart.2006.11.001.

11. Folstein MF, Folstein SE, McHugh PR. "Mini-mental state". A practical method for grading the cognitive state of patients for the clinician. J Psychiatr Res. 1975;12(3):189-98. https://doi.org/10.1016/00223956(75)90026-6.

12. Crum RM. Population-based norms for the mini-mental state examination by age and educational level. JAMA. 1993;269(18):2386-91. https://doi.org/ 10.1001/jama.269.18.2386.

13. Shimizu Y, Yamada S, Suzuki M, et al. Development of the performance measure for activities of daily living-8 for patients with congestive heart failure: a preliminary study. Gerontology. 2010;56(5):459-66. https://doi.org/ 10.1159/000248628.

14. Kono Y, Yamada S, Iwatsu K, et al. Predictive value of functional limitation for disease severity in patients with mild chronic heart failure. J Cardiol. 2012;60(5):411-5. https://doi.org/10.1016/j.jjcc.2012.07.009.

15. Yamada S, Shimizu Y, Suzuki M, Izumi T. Functional limitations predict the risk of rehospitalization among patients with chronic heart failure. Circ J. 2012;76(7):1654-61. https://doi.org/10.1253/circj.CJ-11-1178.

16. Wilson MM, Thomas DR, Rubenstein LZ, et al. Appetite assessment: simple appetite questionnaire predicts weight loss in community-dwelling adults and nursing home residents. Am J Clin Nutr. 2005;82(5):1074-81. 82/5/1074 [pii]

17. Hoyl MT, Alessi CA, Harker JO, et al. Development and testing of a five-item version of the geriatric depression scale. J Am Geriatr Soc. 1999;47(7):873-8. https://doi.org/10.1111/j.1532-5415.1999.tb03848.x

18. McNallan SM, Singh M, Chamberlain AM, et al. Frailty and healthcare utilization among patients with heart failure in the community. JACC Hear Fail. 2013;1(2):135-41. https://doi.org/10.1016/j.jchf.2013.01.002.

19. Valentova M, Von Haehling S, Bauditz J, et al. Intestinal congestion and right ventricular dysfunction: a link with appetite loss, inflammation, and cachexia in chronic heart failure. Eur Heart J. 2016;37(21):1684-91. https://doi.org/10. 1093/eurheartj/ehw008.

20. Shimizu Y, Yamada S, Miyake F, Izumi T. PTMaTCH collaborators. The effects of depression on the course of functional limitations in patients with chronic heart failure. J Card Fail. 2011;17(6):503-10. https://doi.org/10.1016/j. cardfail.2011.01.005.

21. Soysal P, Veronese N, Thompson T, Kahl K. Relationship between depression and frailty in older adults: a systematic review and meta-analysis. Ageing Res. 2017:36:78-87. https://doi.org/10.1016/j.arr.2017.03.005.

22. Lewis EF, Moye LA, Rouleau $J$, et al. Predictors of late development of heart failure in stable survivors of myocardial infarction: the CARE study. J Am Coll Cardiol. 2003;42(8):1446-53. https://doi.org/10.1016/S07351097(03)01057-X

23. Matsuzawa Y, Konishi M, Akiyama E, et al. Association between gait speed as a measure of frailty and risk of cardiovascular events after myocardial infarction. J Am Coll Cardiol. 2013;61(19):1964-72. https://doi.org/10.1016/j. jacc.2013.02.020

24. Sanchis J, Ruiz V, Bonanad C, et al. Prognostic value of geriatric conditions beyond age after acute coronary syndrome. Mayo Clin Proc. 2017;92(6):934-9. https://doi.org/10.1016/j.mayocp.2017.01.018.

25. Kamiya K, Adachi T, Sasou K, Suzuki T, Yamada S. Risk factors for disability progression among Japanese long-term care service users: a 3-year prospective cohort study. Geriatr Gerontol Int. 2017;17(4):568-74. https://doi. org/10.1111/ggi.12756.
26. Dunlay SM, Redfield MM, Weston SA, et al. Hospitalizations after heart failure diagnosis. A community perspective. J Am Coll Cardiol. 2009;54(18):1695702. https://doi.org/10.1016/j.jacc.2009.08.019.

27. Farr BM, Bartlett CLR, Wadsworth J, Miller DL. Risk factors for communityacquire pneumonia diagnosed upon hospital admission. Respir Med. 2000; 94(10):954-63. https://doi.org/10.1053/rmed.2000.0865.

\section{Ready to submit your research? Choose BMC and benefit from:}

- fast, convenient online submission

- thorough peer review by experienced researchers in your field

- rapid publication on acceptance

- support for research data, including large and complex data types

- gold Open Access which fosters wider collaboration and increased citations

- maximum visibility for your research: over $100 \mathrm{M}$ website views per year

At $\mathrm{BMC}$, research is always in progress.

Learn more biomedcentral.com/submissions 